\title{
Contribución de la industria forestal al desarrollo económico de la región del Biobío, Chile
}

\author{
Forest industry contribution to economic development \\ of the Biobio region, Chile
}

\author{
Cristian Mardones P. ${ }^{1}$ y Álvaro Gallardo A. ${ }^{2}$
}

\begin{abstract}
RESUMEN
El objetivo del presente estudio fue evaluar los efectos que tendría un shock exógeno de demanda sobre cada subsector forestal (silvicultura, madera, celulosa y papel y muebles) sobre la actividad económica, remuneraciones, ingreso y medioambiente de la región del Biobío, Chile. Para ello se utilizó una Matriz de Contabilidad Social Extendida Ambientalmente para Biobío con año base 2006 que permitió simular impactos de variaciones en la demanda final con un modelo económico intersectorial. Los resultados permiten concluir que cada subsector forestal genera diferentes contribuciones al desarrollo económico de la región del Biobío. Específicamente, la industria de la madera presenta la más alta participación en el PIB y es la mayor demandante de insumos de otros sectores por su alto encadenamiento hacia atrás, la silvicultura es un sector estratégico, la industria de la celulosa genera mayores efectos en las remuneraciones y altas emisiones contaminantes y la fabricación de muebles genera las menores emisiones contaminantes.
\end{abstract}

PALABRAS CLAVE:

Desarrollo económico, matriz de contabilidad social, medioambiente, modelo insumo-producto.

\begin{abstract}
The aim of this study was to evaluate the effects that would have an exogenous demand shock of forestry sub-sectors (silviculture, timber, pulp and paper, and furniture) on the economic activity, wages, incomes and the environment of the Biobío Region, Chile. For this purpose, was used an Environmental Extended Social Accounting Matrix for Biobío with base year 2006 which allowed simulating impacts of changes in final demand with a sectorial economic model. The results show that each forestry sub-sector generates different contributions to the economic development of the Biobio region, specifically the timber industry has the highest share of GDP and is the greatest consumer of inputs from other sectors for their high backward linkage, silviculture is a strategic sector for its raw material supply, the pulp industry generates greater impact on wages and high pollutant emissions, and furniture manufacturing generates lower pollutant emissions.
\end{abstract}

KEYWORDS:

Economic development, social accounting matrix, environment, input-output model. 


\section{INTRODUCCIÓN}

La economía de la región del Biobío se caracteriza por la importancia de la actividad forestal; según datos del Instituto Nacional de Estadísticas (INE), $72 \%$ de las exportaciones regionales provienen de la industria de la madera, celulosa y papel. En zonas cercanas a la costa de esta región las plantaciones forestales pueden alcanzar participaciones sobre la superficie comunal de hasta $82 \%$. Las especies más importantes son Pinus radiata con $71 \%$ y Eucaliptus globulus con $22 \%$. La producción de madera aserrada regional representa $62 \%$ de la producción nacional, la fabricación de tableros y chapas regional es de $81 \%$ de la producción nacional; además, la región aporta $67 \%$ de la producción de celulosa y el $100 \%$ de la industria de papel del país. A pesar de la relevancia del sector forestal, hasta la fecha no existe un estudio de los impactos que genera esta actividad en el desarrollo económico de la región del Biobío.

Este tipo de análisis cobra relevancia dado que la actividad forestal está fuertemente influenciada por la demanda internacional, por lo que distintos shocks (perturbaciones sobre variables económicas exógenas), que afecten los precios internacionales, tienen repercusiones directas e indirectas en la actividad económica regional. Para analizar estos efectos se utilizan modelos económicos que son capaces de representar el escenario base y escenarios contrafactuales. Una de las herramientas existentes son las matrices de contabilidad social (SAM, Social Accounting Matrix), que representan todas las transacciones y transferencias (monetarias) ocurridas en una economía, en un determinado periodo y nacen de la necesidad de tener un sistema completo ayudando a describir las características estructurales de una economía.

Con una SAM se puede obtener información sobre redistribución del ingreso y patrones de gastos de las instituciones involucradas en las transacciones de bienes y servicios, permitiendo entender las interrelaciones existentes (Keuning Turkey y de Ruijter, 1988). Algunas aplicaciones mencionadas en la literatura son el análisis de la elasticidad del ingreso y los multiplicadores de empleo (Batey y Weeks, 1987); efectos del empleo en la redistribución del ingreso (James y Khan, 1993); análisis de crecimiento económico para países en desarrollo (Vos y Jong, 2003) y análisis de cómo la estructura sectorial del crecimiento contribuye a la desigualdad de ingresos de los hogares, así como entre los trabajadores (Pieters, 2010).

El uso de las SAM también se ha ampliado para agregar variables medioambientales, obteniendo una Matriz de Contabilidad Social Extendida Ambientalmente (SAMEA). Esta matriz extendida provee información integrada al describir las relaciones entre las actividades económicas y las emisiones contaminantes, pero además, puede ser usada como una herramienta de modelación para análisis de políticas relacionadas con la contaminación. Uno de los trabajos pioneros en el desarrollo de estas matrices fue el realizado por Cumberland (1966), extendiendo ambientalmente la matriz para considerar las actividades relacionadas con la contaminación. Se han construido SAMEA para diversos países como China (Xie, 2000), Bolivia (Alarcón et al., 2000), Brasil (Lenzen y Schaffer, 2004), España (Morilla et al., 2005), México (González et al., 2008), entre otros.

Este trabajo utiliza una SAMEA para la región del Biobío desarrollada por Mardones y Saavedra (2011), la cual se complementa con otras fuentes de información para analizar cómo una variación en la demanda final de cada subsector de la industria forestal afectaría directa e indirectamente el resto de los sectores 
económicos de la región del Biobío, el nivel de remuneraciones e ingreso y las emisiones contaminantes al aire $y$ al agua. Los resultados permiten concluir que cada subsector forestal genera distintas contribuciones a cada uno de los elementos que conforman el concepto de desarrollo económico.

\section{OBJETIVOS}

El objetivo general de este estudio es cuantificar los impactos directos e indirectos que genera cada uno de los subsectores forestales sobre el desarrollo económico de la región del Biobío, utilizando un modelo económico multisectorial calibrado con datos que permiten caracterizar el flujo circular de la renta y emisiones contaminantes en la economía de esta región.

Los objetivos particulares son la cuantificación de los impactos específicos sobre la actividad económica regional, la distribución del ingreso y el ambiente.

\section{METODOLOGÍA}

Para desarrollar este trabajo, se utilizó una SAM regional extendida ambientalmente que incluyó 24 sectores productivos, siete factores productivos, cinco hogares representativos de los quintiles de ingreso, un gobierno, el resto del país y el resto del mundo, así como también siete contaminantes emitidos al aire y tres descargados al agua. Los datos de la SAM regional provienen de una investigación previamente publicada, la cual fue combinada con datos económicos intersectoriales de los subsectores forestales a nivel país, provenientes de la Matriz
Insumo-Producto de Chile, esto permitió generar un nivel de desagregación de los sectores productivos de la región del Biobío, acorde con el cumplimiento de los objetivos del presente trabajo. Luego, se calibró con esta base de datos el modelo multisectorial de Pyatt y Round (1979) para realizar análisis contrafactuales que permitieron analizar indicadores de aspectos del desarrollo económico alterados por shocks de demanda final en el sector de silvicultura, madera, celulosa y papel y muebles. A continuación se presentan en forma detallada los aspectos metodológicos.

\section{Representación de la SAM regional}

Una SAM regional refleja las interrelaciones entre los diversos sectores $y$ agentes económicos de una región, pero también las transacciones económicas con el resto del país y el resto del mundo. El grado de desagregación de la SAM depende del objetivo del estudio, en este caso se contaba con una matriz con mayor agregación para el sector forestal que el requerido. Específicamente, la SAM disponible fue elaborada por Mardones y Saavedra (2011). Ésta agregaba la actividad silvícola con la agrícola y la industria maderera con la industria de muebles, por lo cual tuvo que ser desagregada con información complementaria, mediante métodos indirectos de optimización para considerar explícitamente los subsectores forestales de interés: silvícola, industria de la madera, industria de la celulosa y papel e industria de muebles (ver detalles del procedimiento de desagregación en el Apéndice A). Esta nueva SAM incluyó otros 20 sectores ${ }^{1}$ que a través de su interrelación

1 Agropecuario; pesca; minería; alimentos, bebidas y tabaco; textil, prendas de vestir y cuero; química, petróleo, caucho y plástico; fabricación de productos minerales no metálicos; metálica básica; productos metálicos, maquinaria y equipos; resto industria; electricidad; gas; agua; construcción; comercio, restaurantes y hoteles; transporte; comunicaciones; servicios financieros y empresariales; servicios personales y propiedad de vivienda; administración pública. 
económica con los subsectores forestales ayudaron a determinar los efectos indirectos sobre el desarrollo económico. Finalmente, para obtener indicadores más específicos sobre la distribución del ingreso se dividieron los hogares en quintiles de ingreso, el trabajo según género y nivel de estudios (no calificado, semicalificado y calificado). Para ello se utilizó información de la Encuesta de Caracterización Socioeconómica Nacional (CASEN) sobre los ingresos de los hogares de la región del Biobío. Dichos ingresos fueron ordenados, de mayor a menor, en cinco partes iguales al número de hogares, con lo cual se obtuvieron los cinco quintiles (donde el primer quintil representa $20 \%$ de la población más pobre hasta el quinto quintil que representa $20 \%$ de la población más rica). La misma fuente se utilizó para la desagregación de remuneraciones regionales por sector económico y por género, con la cual se construyeron las participaciones relativas sobre las remuneraciones totales de cada sector económico de la SAM.

\section{Matriz de Contabilidad Social y Medioambiental}

De la misma forma que la SAM, la extensión ambiental estuvo sujeta a restricciones de información por los niveles de desagregación requeridos, lo que obviamente afecta la validez de las conclusiones. En este estudio, para obtener las emisiones de contaminación, se utilizó información del Registro Nacional de Emisiones y Transferencias de Contaminantes (RETC) y de la Encuesta Nacional Industrial Anual (ENIA). Para el subsector industria de la madera, celulosa y papel y fabricación de muebles las emisiones en toneladas de material particulado respirable $\left(\mathrm{MP}_{10}\right)$, monóxido de carbono $(\mathrm{CO})$, óxidos de nitrógeno $\left(\mathrm{NO}_{\mathrm{x}}\right)$ y dióxido de azufre $\left(\mathrm{SO}_{\mathrm{x}}\right)$ fueron obtenidas indirectamente de la ENIA. Las emisiones, en toneladas, por tipo de contaminante asociadas a cada sector económico se estimaron a partir de los consumos totales de combustibles, multiplicados por sus respectivos factores de emisión (kg de contaminante / $\mathrm{kg}$ de combustible), siguiendo la metodología del informe "Estimación de emisiones contaminantes atmosféricas a partir de la encuesta nacional industrial anual para alimentar el registro nacional de emisiones y transferencias de contaminantes (RETC)". Para los sectores económicos no industriales, cuyas emisiones no se pudieron obtener de la ENIA, se utilizó información ya procesada del RETC, en la cual se detallaban las toneladas de contaminantes por sector económico.

\section{Modelo Multisectorial}

La SAM constituye un soporte numérico para desarrollar distintos modelos multisectoriales. Existen modelos multisectoriales con distinto grado de complejidad, los modelos lineales se caracterizan por asumir imposibilidad de sustitución en las funciones de producción y rigideces de precios, por lo que no son apropiados para estudiar situaciones de largo plazo. Para subsanar estas limitaciones, cuando se desean hacer simulaciones de políticas económicas a más largo plazo se utilizan modelos de equilibrio general computables, estáticos o dinámicos.

La alternativa escogida para este estudio, por su simpleza y por el experimento contrafactual a desarrollar, fue la utilización del modelo lineal de Pyatt y Round (1979), el cual exige definir las cuentas en endógenas y exógenas. Las cuentas exógenas son las determinadas fuera del sistema económico, constituyendo instrumentos de políticas econó- 
micas y pueden ser las relacionadas con el gobierno, la inversión y el comercio exterior. El resto, por lo tanto, se considera endógeno. Para este trabajo se definieron como cuentas endógenas las ramas de actividad, los factores productivos y los hogares, y como cuentas exógenas el gobierno, inversión, resto del país y resto del mundo. En la tabla 1 se presenta la SAM particionada con base en cuentas endógenas y exógenas.

$\mathrm{N}$ es una matriz de la SAM que representa las transacciones entre las cuentas endógenas, $x$ es una matriz que representa la inyección desde las cuentas exógenas a las endógenas, $L$ es una matriz de fugas de las cuentas endógenas a las exógenas, y $r$ es una matriz de transacciones de la SAM entre las cuentas exógenas. Los multiplicadores de la SAM pretenden visualizar los efectos que se producen en las cuentas endógenas, debido a cambios en las cuentas exógenas, al igual que las Matrices Insumo-Producto, pero la diferencia es que los multiplicadores de la SAM agregan el efecto indirecto e inducido. A continuación se presentan las relaciones de la tabla 1, entre las cuentas endógenas y exógenas:

$$
N=A_{n} \cdot \bar{y}_{n}
$$

La ecuación 1 representa las transacciones entre las cuentas endógenas y es igual a la multiplicación de la matriz de propensiones medias al gasto $A_{n} \mathrm{y}$ el vector de ingresos endógeno.

$$
L=A_{l} \bar{y}_{n}
$$

La ecuación 2 establece que la matriz de fugas $L$ se representa como la multiplicación de la matriz de propensiones medias a las filtraciones $A_{L}$ y el vector de ingresos endógeno.

$$
y_{v}=A_{n} \cdot y_{n}+x
$$

La ecuación 3 muestra que el vector de ingresos endógenos se representa como una suma de dos términos, el primero son las transacciones entre las cuentas endógenas y el segundo las inyecciones desde las cuentas exógenas a las endógenas. Los multiplicadores contables del modelo lineal pueden obtenerse reordenando con algebra matricial la ecuación anterior:

$$
y_{e}=\left(l-A_{n}\right)^{-1} x=M x
$$

donde $M$ es la matriz de multiplicadores contables de la SAM. Cada uno de los elementos de esta matriz, definidos como $m_{i j}$, reflejan el impacto que genera el incremento exógeno en una unidad de ingreso o producción de la cuenta $j$

\begin{tabular}{|c|c|c|c|}
\hline & Cuentas endógenas & Cuentas exógenas & Total \\
\hline Cuentas endógenas & $N=A_{v} \cdot \bar{y}_{n}$ & $x$ & $y_{v}=A_{n} \cdot y_{n}+x$ \\
\hline Cuentas exógenas & $L=A_{L} \cdot \bar{y}_{n}$ & $r$ & $y_{x}=A_{L} \cdot y_{n}+r$ \\
\hline Total & $y_{v}^{\prime}=\left(\mathrm{I}^{\prime} A_{e}+\mathrm{I}^{\prime} A_{2}\right) \cdot \bar{y}_{n}$ & $y_{x}^{\prime}=\Gamma^{\prime} x+\Gamma^{\prime} r$ & \\
\hline
\end{tabular}
(columna $j$ ) sobre el ingreso o producción de la cuenta endógena $i$ (fila $i$ ).

Tabla 1. Interrelación entre cuentas endógenas y exógenas

Fuente: Pyatt y Round (1979) 


\section{Análisis de Multiplicadores con la Matriz de Contabilidad Social Extendida Ambientalmente}

Es muy importante conocer cómo políticas públicas o shocks podrían afectar el crecimiento económico y el medioambiente, es por eso que se recurre a los análisis de multiplicadores con una SAMEA, los cuales dan cuenta del efecto que tienen los shocks externos sobre la economía y el medioambiente. Para ello hay que definir las cuentas que pertenecen a exógenas y endógenas, como se presenta en la tabla 2.

Se pueden relacionar las cuentas medioambientales con las variables económicas en términos físicos, al considerar que las variables medioambientales tienen directa proporcionalidad con la producción de los sectores económicos. Así, se puede estimar un vector de coeficientes técnicos de emisiones al medioambiente $\left(\varepsilon_{i l}\right)$ :

$$
\varepsilon_{d}=C_{d} \cdot y_{i}^{-1}
$$

$C_{i l}$ es un vector de emisiones al medioambiente del contaminante $I$, emitido por el sector $i$, por lo que la ecuación 5 se define como la variación del nivel del contaminante $I$, emitido por el sector $i$, por unidad de producción.
Finalmente, se analizó cómo respondieron las cuentas medioambientales ante variaciones en las cuentas exógenas, por medio de la agregación de los multiplicadores del modelo.

$$
y_{v}=M^{\sin x_{x}}
$$

De esta forma se obtuvieron los indicadores que capturan las variaciones de niveles de los contaminantes, considerando los efectos totales sobre la economía.

$$
\varepsilon_{a}^{D}=\sum_{J} m_{y}^{\mathrm{sims}} \cdot \varepsilon_{a}
$$

\section{RESULTADOS}

Se presentan a continuación los principales resultados, a partir de la obtención de la SAMEA que desagrega el sector forestal para la región del Biobío (ver Apéndice B) y la calibración de un modelo lineal multisectorial que permitió modelar shocks de demanda sobre cada uno de los subsectores forestales analizados.

\section{Participación en el PIB}

Definiendo el PIB sectorial como la suma del pago a remuneraciones, capital, IVA neto y aranceles es posible construir, a

Tabla 2. División de la SAMEA en cuentas endógenas y exógenas

\begin{tabular}{cccccc}
\hline \multicolumn{2}{c}{ SAMEA } & & SAM & & EA \\
\hline & & $\begin{array}{c}\text { Cuentas } \\
\text { endógenas }\end{array}$ & $\begin{array}{c}\text { Cuentas } \\
\text { exógenas }\end{array}$ & Total & $\begin{array}{c}\text { Cuentas } \\
\text { endógenas } \\
\text { medioambientales }\end{array}$ \\
\hline $\mathrm{A}$ & Cuentas endógenas & $N$ & $x$ & $y_{n}$ & $C$ \\
$\mathrm{M}$ & Cuentas exógenas & $L$ & $r$ & $y_{x}$ & - \\
\hline & Total & $y_{n}$ & $y x$ & - & $C T$ \\
\hline
\end{tabular}

Fuente: Adaptado de Morilla et al. (2007) 
partir de los datos de la SAM, una aproximación del aporte de la industria forestal al PIB de la región del Biobío. La mayor participación en el PIB del sector forestal de la región del Biobío pertenece al subsector de industria de la madera $(7,3 \%)$, le sigue el subsector celulosa y papel $(6,6 \%)$, muebles $(1,8 \%) \mathrm{y}$, por último, el subsector silvícola $(1,6 \%)$. Sumando los aportes se concluye que el sector forestal tiene una participación de $17,3 \%$ en el PIB regional, mientras que la participación de estos mismos sectores en el PIB nacional es de aproximadamente $2,6 \%^{2}$, mostrando la importancia de este sector en la economía de esta región.

\section{Indicadores de producción}

El encadenamiento hacia atrás del sector j-ésimo $\left(B L_{j}\right)$ corresponde a la suma de los elementos de la columna $j$ de la matriz (M) de multiplicadores contables de la SAM, mientras el encadenamiento hacia adelante del sector $i$-ésimo $\left(F L_{i}\right)$ corresponde a la suma de los elementos de la fila $i$ de esta matriz.
Un sector con alto encadenamiento hacia atrás es capaz de transmitir los beneficios de cualquier incremento en su demanda final al resto de los sectores. Lo anterior se explica porque para satisfacer este nuevo requerimiento con mayor producción debe, a su vez, demandar insumos de otros sectores productivos que lo abastecen y, a su vez, estos nuevos sectores para elevar su producción requieren demandar insumos de otros sectores, y así sucesivamente hasta que se alcanza la convergencia. Por otra parte, los sectores con alto encadenamiento hacia adelante son aquéllos que elevan fuertemente su producción, estimulados por un incremento la demanda final de todos los sectores económicos, es por ello que son muy sensibles a los cambios que experimente la economía en su conjunto.

En la tabla 3 se observa el encadenamiento hacia atrás de los subsectores forestales de la región del Biobío. Los subsectores de muebles $(2,16)$ y silvicultura $(2,29)$ están bajo la media, mientras los subsectores celulosa y papel $(4,75)$ e industria de la madera $(5,32)$ están sobre la media. Así, este último subsector

Tabla 3. Encadenamientos hacia atrás y hacia adelante

\begin{tabular}{lcc}
\hline Sector & Encadenamiento hacia atrás & Encadenamiento hacia adelante \\
\hline Silvícola & 2,2907 & 3,1396 \\
Madera & 5,3168 & 1,9119 \\
Muebles & 2,1608 & 3,0959 \\
Celulosa y papel & 4,7481 & 2,2630 \\
Media sector forestal & 3,6291 & 2,6026 \\
Media sectorial región del Biobío & 3,9734 & 3,8680 \\
\hline
\end{tabular}

2 Esta información se encuentra agregada en el Banco Central de Chile en los sectores de madera y muebles, agropecuario-silvícola y papel e imprentas. Para el cálculo se utilizan los sectores de madera y muebles, silvícola y papel, desagregándolos con base en el porcentaje de valor agregado de cada sector en la MIP del año 2003. 
forestal, a través de su demanda de insumos, es capaz de transmitir más fuertemente los beneficios de cualquier incremento en su demanda final al resto de los sectores de la economía. En la tabla 3 también se observa el encadenamiento hacia adelante, concluyéndose que los sectores que incrementarían más fuertemente su producción ante un aumento en la demanda final de toda la economía serían en orden decreciente, la silvicultura $(3,14)$, muebles $(3,10)$, celulosa y papel $(2,26)$ y la industria de la madera $(1,91)$.

El indicador de encadenamiento hacia atrás también sirve para simular cómo un shock de demanda de un subsector forestal impactaría sobre la producción de toda la economía regional. En la tabla 4 se ilustra que si la demanda final de cada subsector se incrementara en $1 \%$ del valor de la producción (segunda columna), las sucesivas rondas de la demanda derivada sobre otros sectores productivos que abastecen al sector inicial generaría que la actividad económica regional se incremente tantas veces como el valor del encadenamiento hacia atrás del sector estimulado (tercera columna). Por ejemplo, si el subsector industria de la madera recibe un shock que aumenta su demanda final en el equivalente a $1 \%$ de su producción, es decir, 7239 millones de pesos, dado que el indicador de encadenamiento hacia atrás presentado en la tabla 3 es de 5,32, la producción regional de todos los sectores aumentaría finalmente en 38488 millones de pesos (este valor incluye el aumento de
7239 millones de pesos producto del aumento en la demanda final del sector industria de la madera).

\section{Medidas de dispersión y sectores claves}

Con los encadenamientos hacia atrás y adelante, se identifican los sectores industriales que más impactan sobre la economía, pero también es necesario saber cómo los impactos de un sector se distribuyen o dispersan a través de toda la economía. Puede ocurrir el hecho de que un sector tenga un gran multiplicador, pero pocos sectores se vean afectados, existiendo concentración en el multiplicador. Por lo anterior, utilizando el valor del encadenamiento de cada sector, divido por el valor del encadenamiento promedio sectorial hacia atrás $\left(\pi_{j}=B L, / \overline{B L}\right)$ y hacia adelante $\left(r_{t}=F_{4} / \overline{F L}\right)$ se pueden clasificar los sectores, de acuerdo con Schuschny (2005), como estratégicos $\left(x_{i} \geq 1\right.$ y $\left.x_{j}<1\right)$, claves $\left(t_{i} \geq 1\right.$ y $\left.\pi_{i} \geq 1\right)$, independientes $\left(x_{i}<1\right.$ y $\left.\pi_{i}<1\right)$ o impulsores $\left(x_{i}<1 \quad \mathrm{y} x_{i} \geq 1\right)$.

Con base en los resultados obtenidos, se puede afirmar que en la región los subsectores de industria de la madera ( $\tau_{i}=0,54$ y $\left.\pi_{j}=1,58\right)$ y celulosa y papel ( $\tau_{i}=0,64$ y $\left.\pi_{j}=1,31\right)$ poseen bajos encadenamientos relativos hacia adelante y altos hacia atrás, por lo que son grandes impulsores de la economía, teniendo una elevada oferta de productos que abastecen a la demanda final. El subsector silvícola $\left(\tau_{i}=1,37\right.$ y $\left.\pi_{j}=0,47\right)$ es estratégico

Tabla 4. Impacto total sobre la economía ante un aumento de la demanda sectorial

\begin{tabular}{lcc}
\hline Sector & 1\% de producción $(M M \$)$ & Impacto en la economía $(M M \$)$ \\
\hline Silvícola & 1768 & 4050 \\
Madera & 7239 & 38488 \\
Muebles & 1740 & 3760 \\
Celulosa y papel & 6913 & 32823 \\
\hline
\end{tabular}


ya que no tiene una gran demanda de insumos, pero abastece sustantivamente de insumos a otros sectores, por lo que de él depende abastecer a los sectores que demandan sus insumos. El subsector de muebles $\left(\tau_{i}=0,58\right.$ y $\left.\pi_{j}=0,45\right)$ es un sector independiente, el cual consume pocos insumos intermedios y dedica su producción sustantivamente a satisfacer la demanda final.

\section{Análisis en la distribución de las remuneraciones e ingresos}

En la tabla 5 se presentan los impactos sobre las remuneraciones por niveles de calificación ante la simulación de un aumento de $1 \%$ de la producción de cada subsector forestal. Este efecto sobre los ingresos laborales $\left(I_{j}\right)$ se calcula como la suma multiplicadores contables del modelo $m_{i j}$ ponderado por la participación del pago al trabajo sobre el valor de la producción del sector $i\left(w_{i}\right)$, es decir, $J_{3}-\sum \mathrm{m}_{2} \boldsymbol{\pi}_{\mathrm{s}}$. Por lo anterior, este indicador representa el incremento en las remuneraciones generado en toda la economía regional luego del shock inicial de demanda en un subsector forestal particular.

Tal como se aprecia en la tabla 5 si ocurre un shock de demanda en el sector de la primera columna equivalente a $1 \%$

Tabla 5. Efecto en las remuneraciones ante un aumento de $1 \%$ en la demanda (MM\$)

\begin{tabular}{lllccc}
\hline Sector & Nivel de & $\begin{array}{c}1 \% \text { de } \\
\text { calificación }\end{array}$ & $\begin{array}{c}\text { Impacto } \\
\text { directo }\end{array}$ & $\begin{array}{c}\text { Impacto } \\
\text { indirecto }\end{array}$ & $\begin{array}{c}\text { Impacto } \\
\text { total }\end{array}$ \\
\hline \multirow{3}{*}{ Silvícola } & Hombre no calificado & 1768 & 46 & 46 & 91 \\
& Hombre semicalificado & 1768 & 38 & 47 & 85 \\
& Hombre calificado & 1768 & 33 & 48 & 81 \\
& Mujer no calificada & 1768 & 52 & 47 & 99 \\
\multirow{5}{*}{ Madera } & Mujer semicalificada & 1768 & 22 & 52 & 74 \\
& Mujer calificada & 1768 & 17 & 57 & 74 \\
& Hombre no calificado & 7239 & 548 & 662 & 1211 \\
& Hombre semicalificado & 7239 & 524 & 666 & 1190 \\
& Hombre calificado & 7239 & 393 & 631 & 1024 \\
Muebles & Mujer no calificada & 7239 & 253 & 640 & 893 \\
& Mujer semicalificada & 7239 & 123 & 683 & 806 \\
& Mujer calificada & 7239 & 84 & 729 & 813 \\
& Hombre no calificado & 1740 & 61 & 42 & 103 \\
& Hombre semicalificado & 1740 & 37 & 41 & 79 \\
& Hombre calificado & 1740 & 19 & 40 & 60 \\
& Mujer no calificada & 1740 & - & 41 & 41 \\
& Mujer semicalificada & 1740 & 17 & 44 & 61 \\
& Mujer calificada & 1740 & - & 46 & 46 \\
& Hombre no calificado & 6913 & 177 & 568 & 745 \\
& Hombre semicalificado & 6913 & 260 & 594 & 854 \\
& Hombre calificado & 6913 & 408 & 557 & 965 \\
& Mujer no calificada & 6913 & 200 & 543 & 742 \\
& Mujer semicalificada & 6913 & 220 & 604 & 823 \\
& Mujer calificada & 6913 & 137 & 636 & 773 \\
\hline & & & & &
\end{tabular}


de su producción, representado en la tercera columna, para cada tipo de mano de obra (segunda columna) se provocaría un incremento directo sobre las remuneraciones por la mayor contratación en el sector, afectado por el alza inicial de la demanda (cuarta columna). Pero además, este sector demandaría insumos de otros sectores con los que tenga encadenamientos productivos. Esto elevaría, en una segunda ronda, las remuneraciones de los trabajadores de esos sectores. Esta demanda inducida se sigue repitiendo en sucesivas rondas y elevando nuevamente las remuneraciones, hasta que se alcanza la convergencia; por lo que a la suma de estos sucesivos efectos se les define como efectos indirectos (quinta columna), los cuales sumados al efecto directo arrojan el efecto final sobre las remuneraciones. A modo de ejemplo, si aumenta la demanda en la industria de la madera 1\% (7 239 millones), la producción debe expandirse al contratar y pagar a la mano de obra no calificada masculina 548 millones de pesos por impacto directo. Pero además, los impactos a través de los encadenamientos productivos sobre el resto de los sectores económicos de la región (que también ven elevadas sus demandas, producciones y contrataciones de mano de obra de forma inducida) elevan adicionalmente el pago de remuneraciones para el nivel no calificado masculino en 662 millones de pesos. Por lo que el efecto total es de 1211 millones de pesos.
La mayor diferencia entre impactos directos e indirectos ocurre para el subsector de industria de la madera, nivel calificado femenino, que además posee el mayor impacto indirecto. Por otro lado, el mayor impacto directo corresponde al subsector de industria de la madera, nivel no calificado masculino, que además posee el mayor impacto total.

Para analizar los cambios sobre la distribución del ingreso asociados a un shock de demanda se utilizan los multiplicadores contables del modelo $m_{i j}$ (mostrados en la tabla 6), donde el subíndice $i$ representa el quintil de ingreso y el subíndice $j$ representa el sector afectado por un shock de demanda. Al calcular la participación relativa de estos multiplicadores por cada subsector forestal, se obtiene que en la silvicultura la mayor parte de los ingresos generados van al quintil $1(22,3 \%)$ y la menor cantidad van al quintil 5 (17,9\%); para la industria de la madera la mayor parte de los ingresos generados van al quintil $4(20,6 \%)$ y la menor cantidad al quintil $1(19,5 \%)$; mientras que para la fabricación de muebles la mayor parte de los ingresos generados van al quintil $1(22,2 \%)$ y la menor cantidad al quintil $5(17,7 \%)$. Por último, para la industria de celulosa y papel, la mayor parte de los ingresos generados van al quintil $5(22,1 \%)$ y la menor cantidad van al quintil $1(17,6 \%)$.

Tabla 6. Multiplicadores contables del modelo para los quintiles de ingreso

\begin{tabular}{lccccc}
\hline Sector & Quintil 1 & Quintil 2 & Quintil 3 & Quintil 4 & Quintil 5 \\
\hline Silvícola & 0048 & 0047 & 0045 & 0044 & 0043 \\
Madera & 0150 & 0151 & 0154 & 0158 & 0155 \\
Muebles & 0045 & 0044 & 0041 & 0039 & 0036 \\
Celulosa y papel & 0113 & 0120 & 0130 & 0138 & 0142 \\
\hline
\end{tabular}




\section{Análisis de impactos ambientales}

En la tabla 7 se presenta el efecto, según las emisiones en miles de toneladas, de un aumento de $1 \%$ en la demanda final de cada uno de los subsectores forestales de la región del Biobío. El efecto directo se produce por el incremento de las emisiones necesarias para elevar la producción ante el shock de demanda, pero el efecto indirecto se genera por el incremento en la actividad y emisiones inducidas en el resto de los sectores económicos de la región.

A pesar de que los subsectores silvícola y fabricación de muebles casi no generan emisiones directamente, por el efecto indirecto que estimula la demanda sobre el resto de los sectores económicos, eleva sus emisiones totales. Se observa también que aún cuando la industria maderera genera directamente mucho menos emisiones que la industria de celulosa y papel, tal como se podía esperar, la emisiones indirectas generadas por los otros sectores estimulados a través de la demanda de insumos (por ejemplo transporte, energía, entre otros) luego del shock de demanda inicial, hace finalmente que sean bastante semejantes las emisiones totales al aire de ambos sectores.

\section{DISCUSIÓN}

Los resultados generados a partir de la metodología empleada aportan información valiosa sobre las interrelaciones del sector forestal con el resto de la economía, la distribución de los ingresos y el medioambiente en la región. De esa forma es que se pueden entender los canales que transmiten los shocks económicos asociados a la actividad forestal y, además, su compresión ayuda al diseño de políticas económicas que afecten a este sector y su repercusión en los distintos componentes del desarrollo económico. Por ejemplo, políticas públicas de fomento para la adquisición de muebles ayuda a mejorar la distribución del ingreso y genera poca contaminación en términos directos e indirectos, pero difícilmente genera círculos virtuosos de crecimiento en otros sectores, dado su escaso encadenamiento productivo. La continuación de políticas de fomento a las plantaciones forestales, tal como las que actualmente existen en Chile, a pesar de no generar mejoras sustantivas en el PIB, contribuyen estratégicamente para que se desarrollen otros sectores como la industria de la madera y la industria de la celulosa y papel.

En esta línea de análisis, la caracterización del sector forestal realizada en este estudio puede ayudar a entender las dinámicas económicas en otras regiones y países, por lo que a continuación se presenta una breve discusión de cada subsector forestal.

El sector silvícola tiene una participación en el PIB regional de 1,6\%, su producción se dirige principalmente a uso intermedio, por lo que es un sector estratégico. Si su demanda aumenta en $1 \%$, sería el penúltimo subsector forestal en tener un impacto en la economía por encadenamientos hacia atrás y hacia delante, pero además produciría la segunda menor desigualdad en la distribución de los ingresos generados según quintiles. También es el sector que más aporta en términos relativos a la equidad de género con las remuneraciones de la mano de obra no calificada, aunque con montos no muy significativos. Las emisiones directas al aire y agua son marginales, pero las indirectas son relevantes y muy similares a las generadas por la fabricación de muebles. 


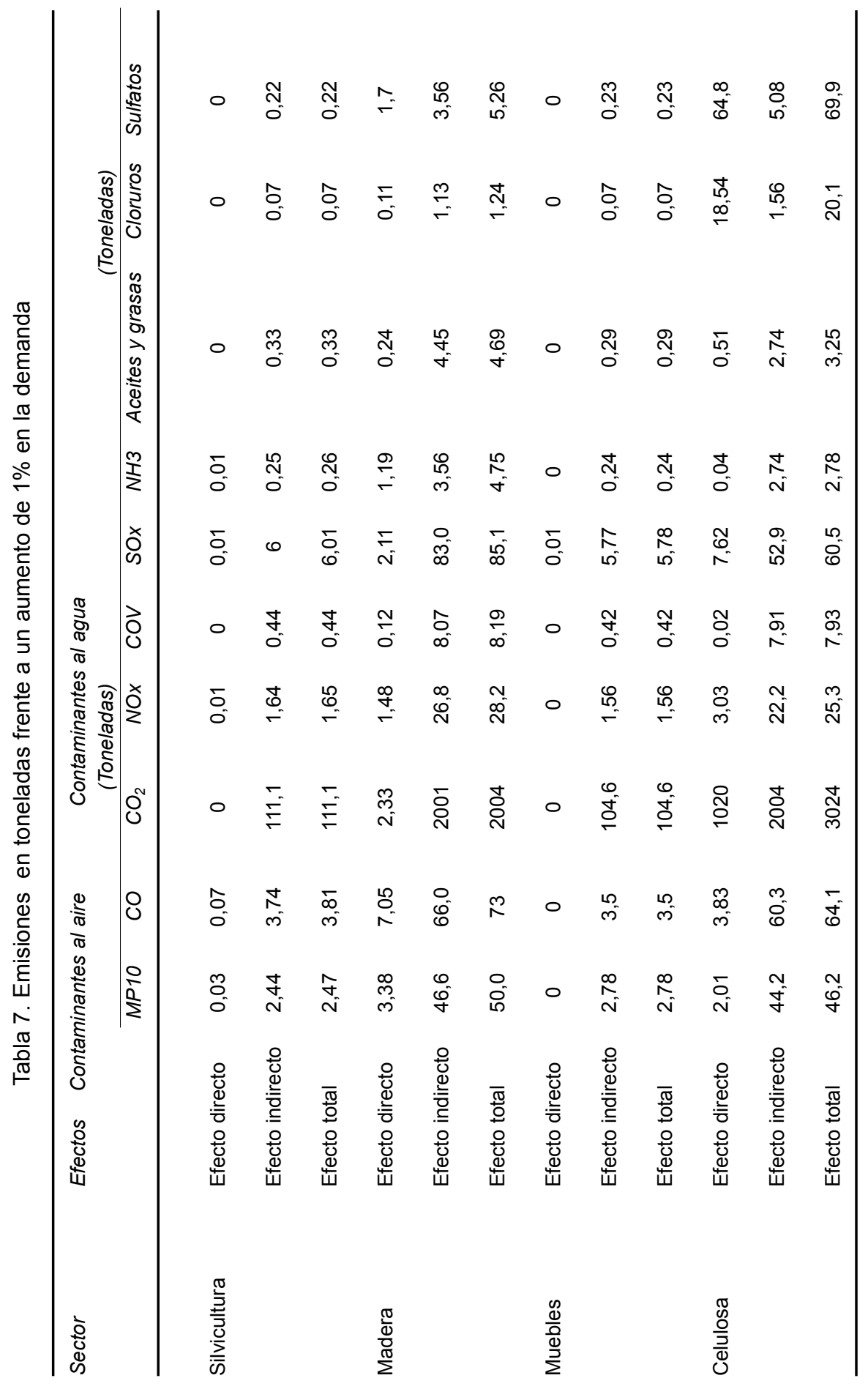


El sector de la industria de la madera tiene una participación en el PIB regional de $7,3 \%$, su producción se dirige en gran medida a la demanda final. Tiene el mayor impacto en la economía por encadenamientos hacia atrás y el último por encadenamientos hacia adelante. También es un gran impulsor de la economía con productos que abastecen a la demanda final. Por otro lado, posee el mayor impacto sobre las remuneraciones para todos los niveles de calificación, generando la segunda mayor desigualdad en la distribución de los ingresos por quintiles, pero incrementa de forma importante las brechas de las remuneraciones por género. Tiene las mayores emisiones totales de $\mathrm{MP}_{10}, \mathrm{CO}, \mathrm{NO}_{\mathrm{x}}$, $\mathrm{SO}_{\mathrm{x}}$, y $\mathrm{NH}_{3}$, aceites y grasas, ante un aumento de $1 \%$ en su demanda.

La industria de la celulosa y papel tiene una participación en el PIB regional de $6,6 \%$. Es el segundo subsector con mayor impacto en la economía por encadenamientos hacia atrás y el penúltimo por encadenamientos hacia delante. Es un gran impulsor de la economía al abastecer a la demanda final. Además, ante un shock de demanda, el ingreso distribuido al quintil 5 representa 1,3 veces el ingreso del quintil 1, generando la mayor desigualdad entre hogares, aunque los aportes a las remuneraciones de la mano de obra femenina son significativas. Este subsector tiene las mayores emisiones totales de $\mathrm{CO}_{2}$, cloruros y sulfatos y el segundo lugar en otros contaminantes ante un aumento de $1 \%$ en la demanda.

El subsector de fabricación de muebles tiene una participación en el PIB regional de $1,8 \%$. Es el subsector que tendría un impacto menor en la economía por encadenamientos hacia atrás, pero el segundo por encadenamientos hacia delante. El subsector de fabricación de muebles consume pocos insumos intermedios y dedica su producción sustantivamente a satisfacer la demanda final, por lo que es un sector estratégico. Además, ante un shock de demanda genera la mejor distribución del ingreso, pero aporta negativamente en términos relativos a la equidad de género con las remuneraciones de la mano de obra no calificada, aunque con montos no muy significativos. Tiene las menores emisiones totales de $\mathrm{CO}, \mathrm{CO}_{2}, \mathrm{NO}_{\mathrm{x}}, \mathrm{COV}$, $\mathrm{SO}_{\mathrm{x}}$ y $\mathrm{NH}_{3}$, en relación con los contaminantes al agua, tiene las menores emisiones de aceites y grasas y cloruros, ante un aumento de $1 \%$ de la demanda.

Como discusión final se plantea que la modelación utilizada está especialmente orientada a la evaluación de corto y mediano plazo, dado que en este lapso la estructura productiva es relativamente constante, por lo cual los supuestos son válidos. Sin embargo, si se quisiera estudiar la relación dinámica entre el crecimiento, ingreso de los hogares y emisiones contaminantes, se requeriría la utilización de herramientas más sofisticadas como un modelo regional de equilibrio general computable. ${ }^{3}$

\section{CONCLUSIONES}

Este estudio muestra la utilidad de contar con información regional actualizada para el análisis de distintos sectores económicos y su contribución hacia los distintos componentes del desarrollo económico. Específicamente, si la autoridad de gobierno cuenta con herramientas como las matrices de contabilidad social extendidas ambientalmente para aplicar 
modelos económicos multisectoriales, en función de las prioridades o ponderaciones sobre el crecimiento, distribución del ingreso y medioambiente, será posible el fomento de ciertos sectores en función de sus preferencias u objetivos de políticas.

En el caso de estudio cada uno de los cuatro subsectores forestales generan distintas contribuciones al crecimiento económico regional, a la distribución del ingreso y a la contaminación ambiental, pero lo más importante es que se ha podido cuantificar la importancia de cada uno de estos aportes a la economía de la región del Biobío.

\section{REFERENCIAS}

Alarcón, J., J. van Heemst y N. de Jong. 2000. Extending the SAM with social and environmental indicators: An Application to Bolivia. Economic Systems Research 12(4):473-496.

Batey, P.W.J. y M.J. Weeks. 1987. An extended input-output model incorporating employed, unemployed, and inmigrant households. Papers of the Regional Science Association 62:93-115.

Cumberland, J. 1966. A regional interindustry model for analysis of development objectives. Papers and Proceedings, Regional Science Association 17:65-94.

Fofana, I., A. Lemelin y J. Cockburn. 2002. Balancing a social accounting matrix. Centre de Recherche en Économie et Finances Appliquées (CREFA) Université Laval, October 2002.
González, A. R., G. A. Matus y G. M. González. 2008. Efecto de las políticas económicas en los recursos naturales y el medio ambiente en México. Agrociencia 42(7):847-855.

James, J. y H.A. Khan. 1993. The employment effects of an income redistribution in developing countries. World Development 21(5):817-827.

Lenzen, M. y R. Schaffer. 2004. Environmental and social accounting for Brazil. Environmental and Resource Economics 27:201-226.

Mardones, C. y J. Saavedra. 2011. Matriz de contabilidad social extendida ambientalmente para análisis económico de la región del Biobío. Revista de Análisis Económico 26(1):17-51.

Mardones, C. 2012. Chile: building a computable general equilibrium model with an application to the Biobío region. CEPAL Review 106:125-150.

Morilla, C.R., G. Llanes. y M. A. Cardenete. 2005. Estimación y actualización anual de matrices de contabilidad social: aplicación a la economía española para los años 1995 y 1998. Estadística Española 47(159):353-416.

Partridge, M. y D. Rickman. 2010. Computable general equilibrium (CGE) modelling for regional economic development analysis. Regional Studies 44(10):1311-1328.

Pieters, J. 2010. Growth and inequality in India: analysis of an extended social accounting matrix. World Development 38(3):270-81. 
Pyatt G. y J. Round. 1979. Accountig and fixed price multipliers in a social accounting matrix framework. The Economic Journal 89:850-873.

Schuschny, A. 2005. Tópicos sobre el Modelo de Insumo-Producto: teoría y aplicaciones. CEPAL, Serie Estudios Estadísticos y Prospectivos 37:1-96.
Vos, R y N. Jong. 2003. Trade Liberalization and Poverty in Ecuador: a CGE Macromicrosimulation Analysis. Economic Systems Research (15):211-232.

Xie, J. 2000. An environmentally extended social accounting matrix. Environmental \& Resource Economics 16(4):391-406. Biobío, Chile. Madera y Bosques 18(2):27-50. 


\section{APÉNDICE A. \\ DESAGREGACIÓN Y BALANCEO DE LA SAM}

Para poder desagregar una SAM, cuando no se dispone de esta información a nivel regional, una opción es obtener la información de la Matriz Insumo Producto (MIP) de Chile y/o utilizar información regional no conciliada que proviene de diversas encuestas como la ENIA, CASEN, entre otras. Bajo la primera limitación se considera el supuesto de que la estructura productiva regional utiliza la misma intensidad de uso de insumos que a nivel nacional, para analizar cómo opera este supuesto se presenta un extracto de la MIP de Chile 2003 (la cual posee un grado de desagregación de 73 sectores económicos) en el cuadrante de utilización intermedia total.

Tabla A1. Extracto de la Matriz Insumo Producto de Chile, 2003

\begin{tabular}{lrrrrr}
\hline & \multicolumn{2}{c}{ Agropecuario (\$) } & Silvícola (\$) \\
& & Agricultura & Fruticultura & Ganadería & Silvicultura \\
\hline Agropecuario & Agricultura & 46433 & 3742 & 137429 & 2550 \\
& Ganadería & 24 & 3543 & 15553 & 2022 \\
Silvícola & & & & \\
& & 3 & 92 & 1578 & 296318 \\
\hline
\end{tabular}

FUENTE: MIP 2003. Cantidades en millones de pesos de 2003.

A continuación se presentan los porcentajes de las relaciones entre estas cuentas agregadas.

Tabla A2. Participación de cuentas agregadas

\begin{tabular}{lcc}
\hline & Agropecuario (\%) & Silvicultura (\%) \\
\hline Agropecuario & $40,52 \%$ & $1,05 \%$ \\
Silvicultura & $0,32 \%$ & $58,09 \%$ \\
\hline
\end{tabular}

Estos porcentajes se utilizan para desagregar la SAM del año 2006 para la región del Biobío. En la SAM Regional del año 2006 de Mardones y Saavedra (2011), el sector agropecuario-silvícola compró insumos al sector agropecuario-silvícola por 44635 millones de pesos, pero para el presente estudio se necesitó desagregar ese valor en el sector agropecuario y en el sector silvícola. A continuación se presenta este valor desagregado utilizando los porcentajes expuestos anteriormente.

Tabla A3. Participación de cuentas agregadas

\begin{tabular}{lcc}
\hline & Agropecuario (\$) & Silvicultura (\$) \\
\hline Agropecuario & 18089 & 469 \\
Silvicultura & 146 & 25929 \\
\hline
\end{tabular}


Así, se ha desagregado el sector agropecuario y silvícola en la nueva SAM. Este procedimiento se utiliza iterativamente en el resto de las cuentas relacionadas con el sector agropecuario y luego para el sector silvícola con cada una las restantes cuentas de la matriz.

Otras cuentas de la SAM original que fueron desagregadas fue el sector de madera y muebles, electricidad-gas-agua, así como también el factor trabajo que se desagregó por género y nivel de calificación con base en la encuesta CASEN $2006 .{ }^{4}$

La SAM, luego de su desagregación, necesita que los flujos de ingresos sean iguales a los gastos, por lo que requiere ser balanceada (igualdad de los totales fila y columna). Como solución a los problemas planteados anteriormente, se necesita conocer la matriz cuadrada T de transacciones de la SAM, donde sus celdas tij satisfacen lo siguiente:

$$
t_{i, j}=\sum t_{0}
$$

Luego se obtiene una matriz A de coeficientes técnicos, a partir de la matriz T, cumpliendo cada celda la siguiente restricción:

$$
a_{y}=t_{j} / t_{j}
$$

El método de minimización de distancias o crossentropy parte de una SAM existente, para luego encontrar una nueva SAM, muy cercana a la inicial, por medio de la minimización de la distancia de entropía cruzada entre ellas, respetando todas las restricciones. En el caso del balanceo, la restricción es que el total fila sea igual al total columna. A continuación se presenta el problema de minimización expuesto por Fofana et al. (2002):

$$
\min _{i !} H=\sum \sum t_{j}^{1} \ln \frac{t_{y}^{1}}{t_{s}^{0}}=\sum \sum_{j} t_{y}^{1} \ln t_{y}^{1}-\sum \sum_{j} t_{y}^{1} \ln t_{y}^{a}
$$

Sujeto a:

$$
\begin{gathered}
\sum_{j} t_{y}^{\prime} X_{j}=X_{j} \\
\sum_{j} t_{y}^{\prime}=1
\end{gathered}
$$

Donde $t_{\partial}^{\prime}$ es un nuevo valor de la celda $i j$ y $\quad 0 \leq t_{\partial}^{\prime} \leq 1$. Tanto más cercano esté el valor al valor inicial, la expresic $\ln \frac{f_{y}^{\prime}}{f_{y}} \quad$ va a tender a 0 , minimizado $H$.

4 Se utilizó la siguiente desagregación en la CASEN 2006: No calificado: sin educación formal, básica incompleta, básica completa. Semi-calificado: media humanista incompleta, media técnico profesional incompleta, media humanista completa y medio técnico completo. Calificado: técnica o universitaria incompleta y técnica o universitaria completa. Lo mismo se ocupa para los otros subsectores. 


\section{APÉNDICE B. \\ REPRESENTACIÓN DE LA SAM AGREGADA DE LA REGIÓN DEL BIOBÍO AÑO 2006, EN MILLONES DE PESOS}

Tabla B1. Abreviaturas usadas en SAM agregada y la matriz de multiplicadores

\begin{tabular}{|c|c|c|c|}
\hline & $\begin{array}{l}\text { Actividades } \\
\text { primarias } \\
\text { (prim) }\end{array}$ & $\begin{array}{l}\text { agro } \\
\text { pesca } \\
\text { miner }\end{array}$ & $\begin{array}{l}\text { Agropecuario } \\
\text { Pesca } \\
\text { Minería }\end{array}$ \\
\hline \multicolumn{4}{|c|}{ Actividades Productivas } \\
\hline & $\begin{array}{l}\text { Bienes } \\
\text { (bienes) }\end{array}$ & $\begin{array}{l}\text { alimen } \\
\text { textil } \\
\text { quimic } \\
\text { nomet } \\
\text { metbas } \\
\text { metmec } \\
\text { rindus } \\
\text { elec } \\
\text { gas } \\
\text { agua } \\
\text { constr }\end{array}$ & $\begin{array}{l}\text { Alimentos, bebidas y tabaco } \\
\text { Textil, prendas de vestir y cuero } \\
\text { Química, petróleo, caucho y plástico } \\
\text { Fab.de productos minerales no metálicos } \\
\text { Metálica básica } \\
\text { Productos metálicos, maquinaria y equipos } \\
\text { Resto industria } \\
\text { Electricidad } \\
\text { Gas } \\
\text { Agua } \\
\text { Construcción }\end{array}$ \\
\hline & $\begin{array}{l}\text { Comercio y } \\
\text { servicios } \\
\text { (comyss) }\end{array}$ & $\begin{array}{l}\text { comer } \\
\text { transp } \\
\text { comun } \\
\text { ssfin } \\
\text { srvprop } \\
\text { admpub }\end{array}$ & $\begin{array}{l}\text { Comercio, restaurantes y hoteles } \\
\text { Transporte } \\
\text { Comunicaciones } \\
\text { Servicios financieros y empresariales } \\
\text { Servicios personales y propiedad } \\
\text { de vivienda } \\
\text { Administración pública }\end{array}$ \\
\hline & $\begin{array}{l}\text { Silvicultura } \\
\text { Madera } \\
\text { Celulosa y papel } \\
\text { Muebles }\end{array}$ & $\begin{array}{l}\text { silv } \\
\text { mader } \\
\text { celul } \\
\text { mueble }\end{array}$ & $\begin{array}{l}\text { Silvícola } \\
\text { Madera } \\
\text { Celulosa y papel } \\
\text { Muebles }\end{array}$ \\
\hline Factor Capital & cap & & Factor Capital \\
\hline Factor Trabajo & $\begin{array}{l}\text { nocalif-hom } \\
\text { secalif-hom } \\
\text { calif-hom } \\
\text { nocalif-muj } \\
\text { secalif-muj } \\
\text { calif-muj }\end{array}$ & & $\begin{array}{l}\text { Personal no calificado masculino } \\
\text { Personal semi-calificado masculino } \\
\text { Personal calificado masculino } \\
\text { Personal no calificado femenino } \\
\text { Personal semi-calificado femenino } \\
\text { Personal calificado femenino }\end{array}$ \\
\hline Hogares & $\begin{array}{l}\text { q1 } \\
\text { q2 } \\
\text { q3 } \\
q 4 \\
\text { q5 }\end{array}$ & & $\begin{array}{l}\text { Quintil } 1 \\
\text { Quintil } 2 \\
\text { Quintil } 3 \\
\text { Quintil } 4 \\
\text { Quintil } 5\end{array}$ \\
\hline Impuestos & $\begin{array}{l}\text { instax } \\
\text { acttax } \\
\text { vatax } \\
\text { imptax }\end{array}$ & & $\begin{array}{l}\text { Impuesto directo a la renta } \\
\text { Impuestos indirectos a actividades } \\
\text { Impuesto al valor agregado } \\
\text { Aranceles de importación }\end{array}$ \\
\hline
\end{tabular}




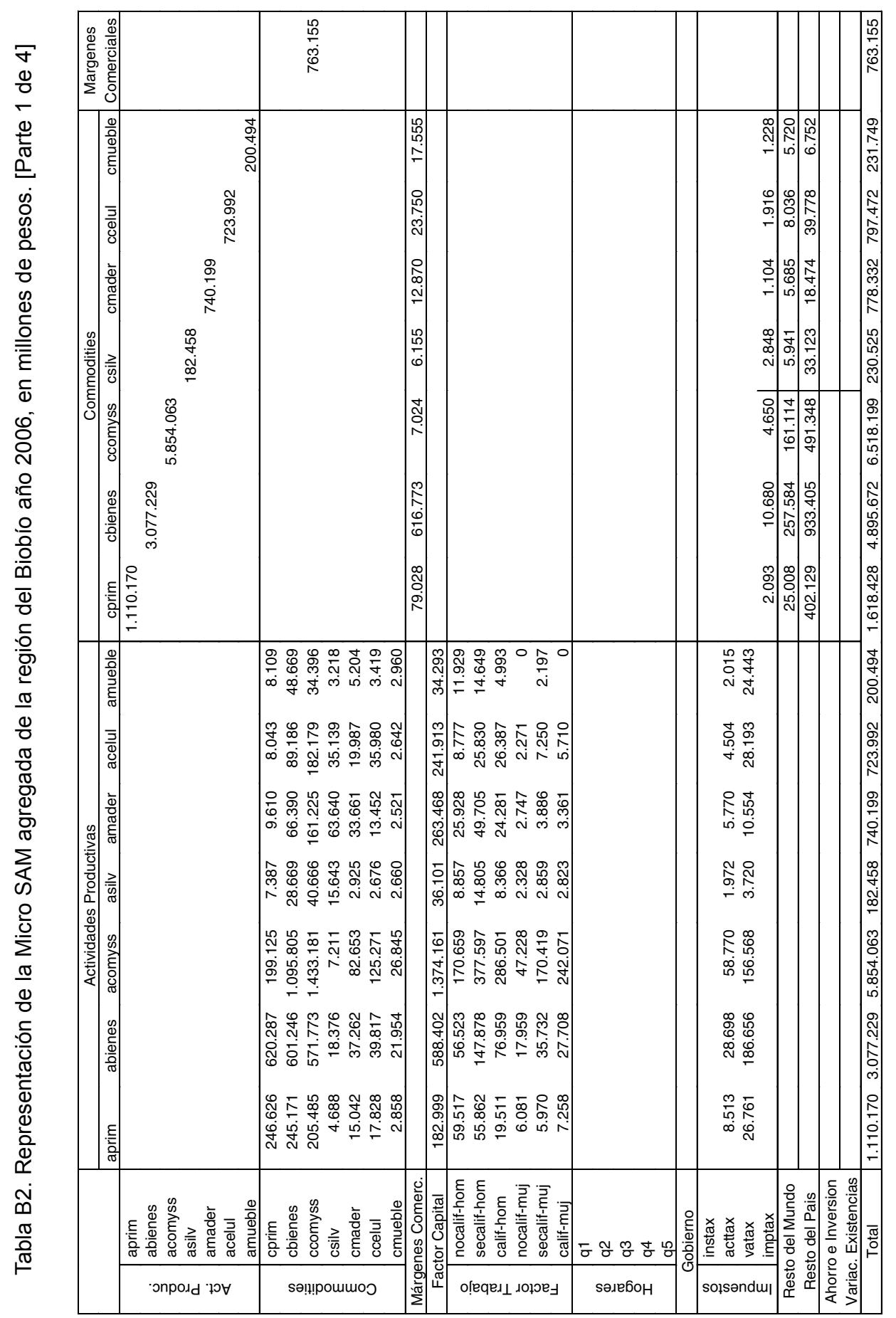




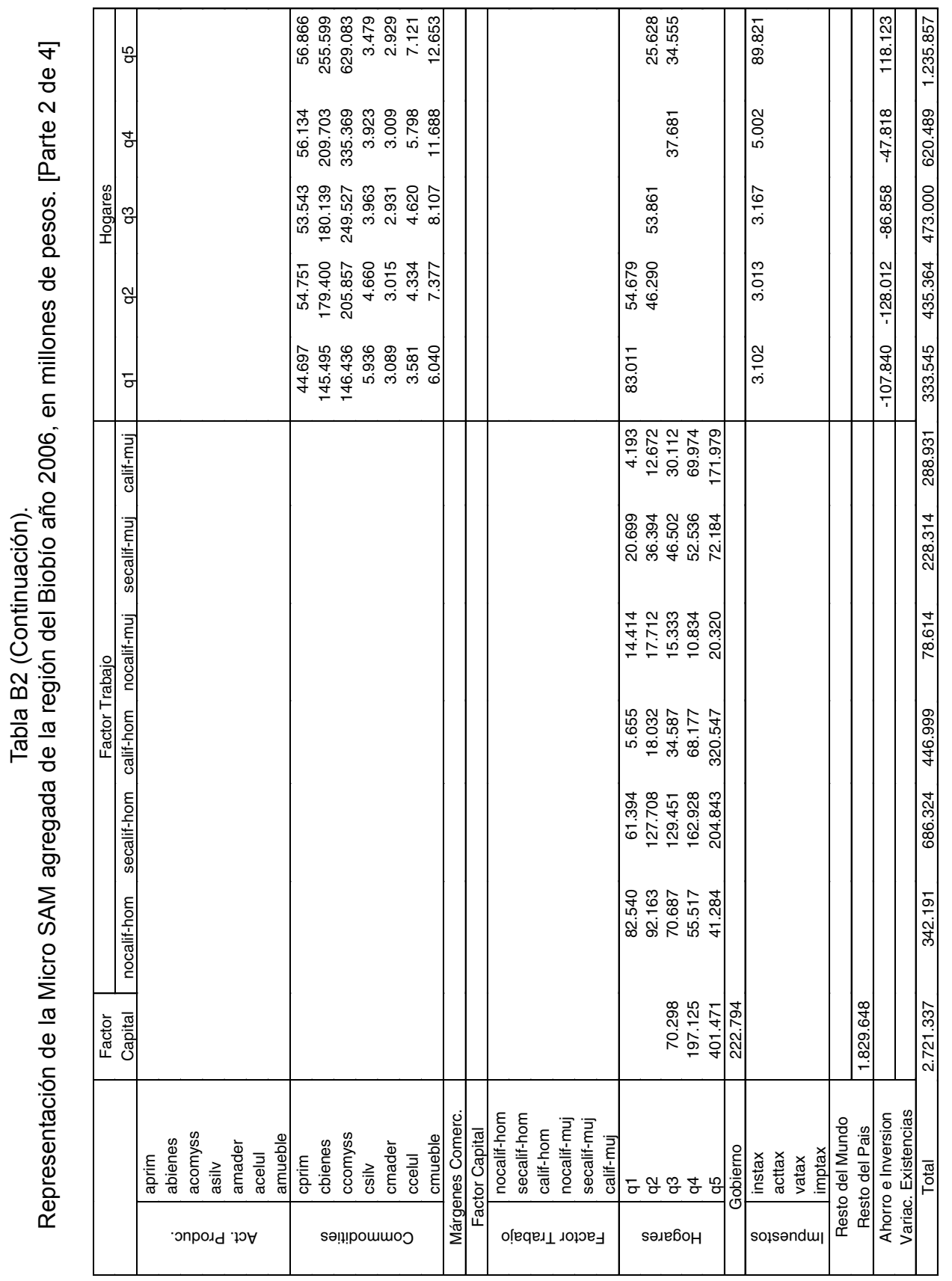




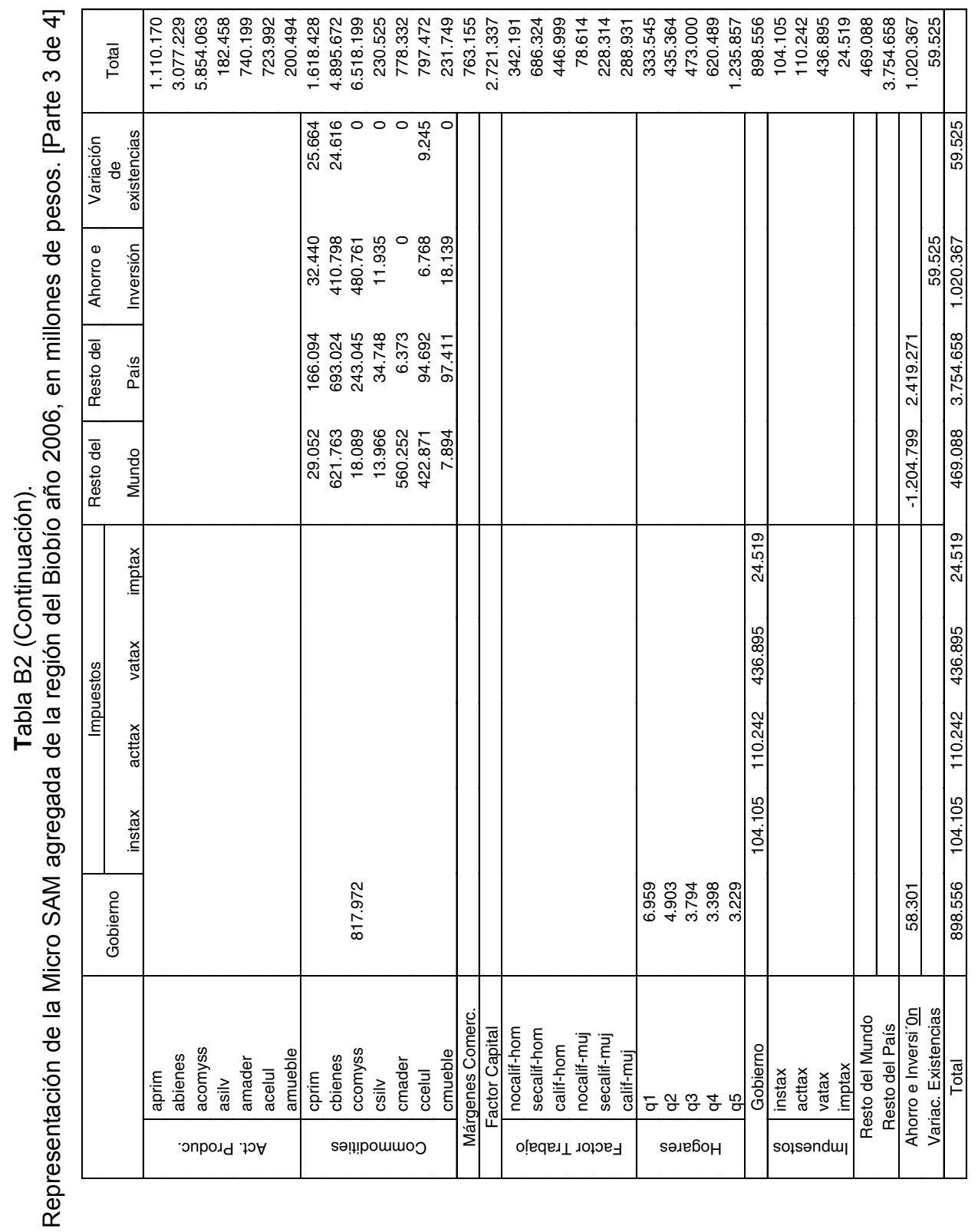




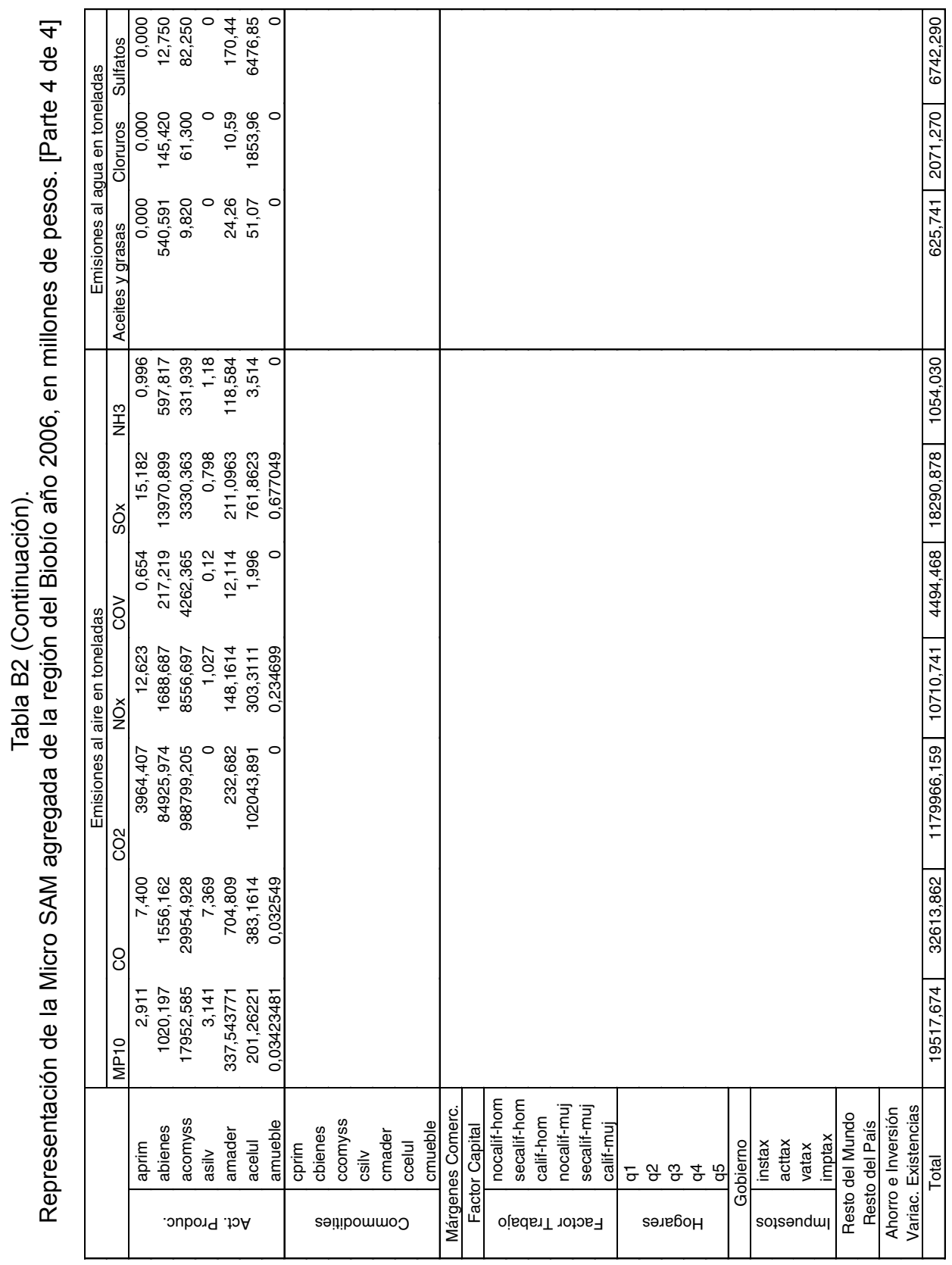




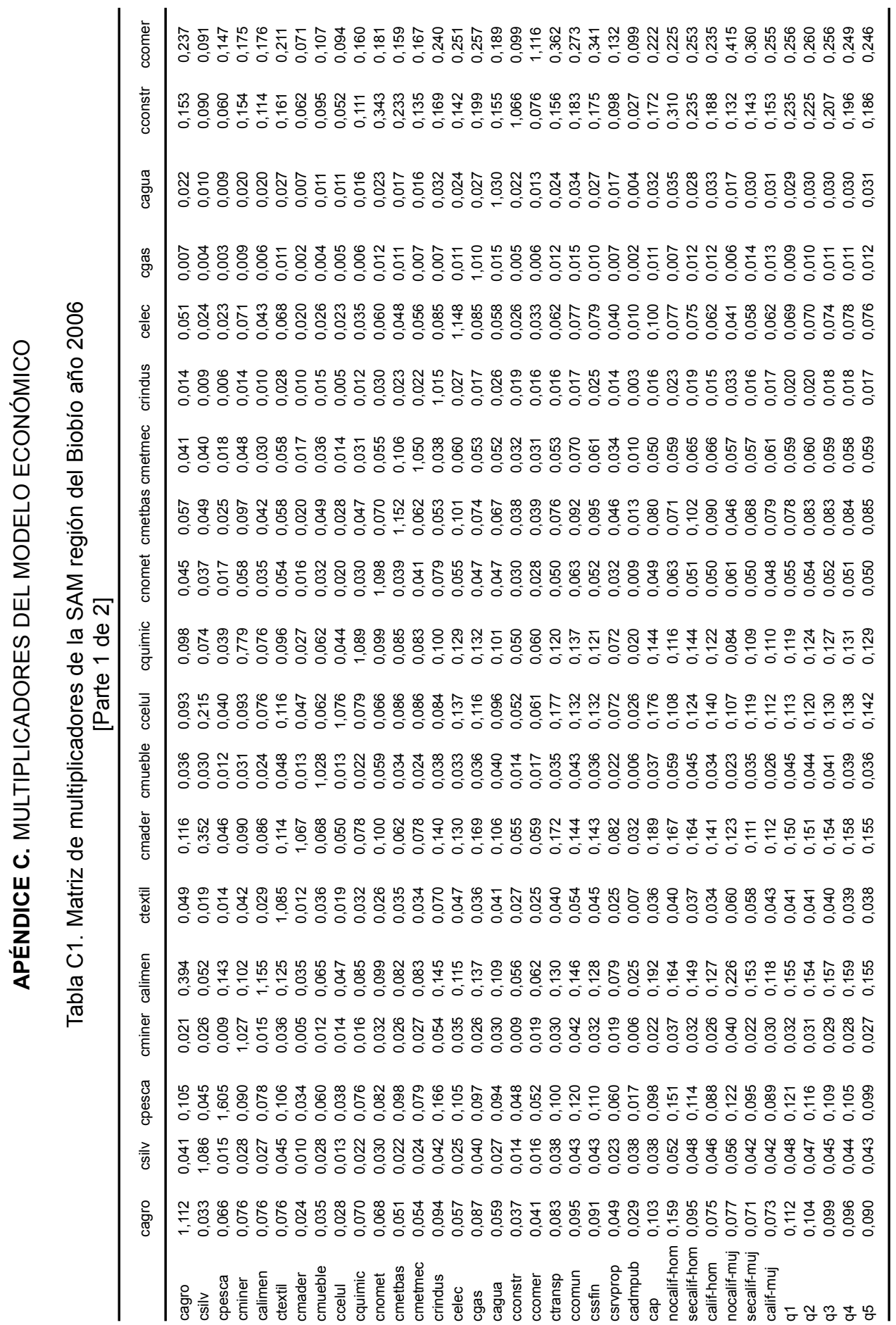




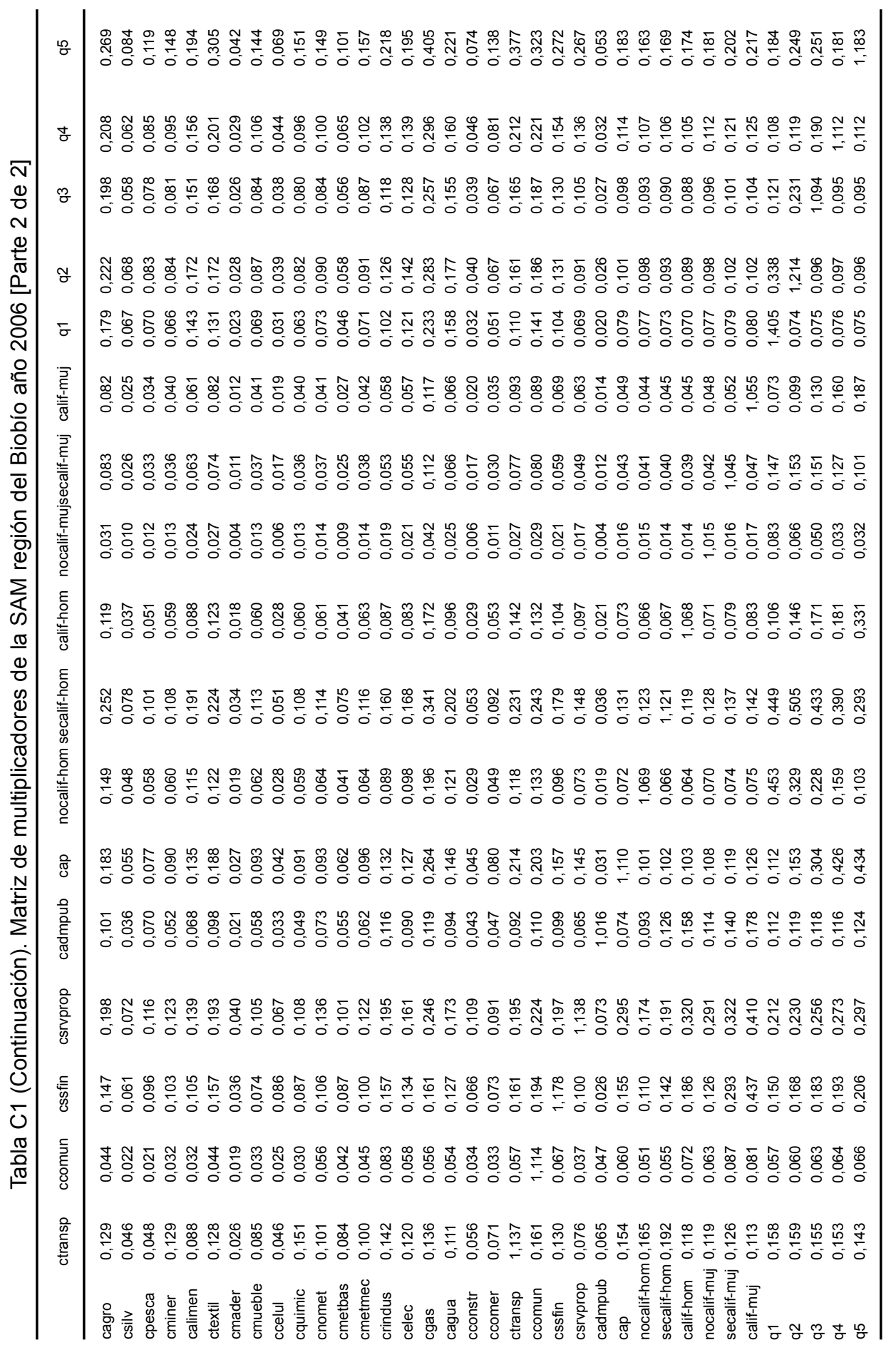

